\title{
Bilateral renal artery variation
}

\author{
Hülya Üçerler ${ }^{1}$, Yusuf Üzüm², Z. Aslı Aktan İkiz ${ }^{1}$ \\ ${ }^{1}$ Department of Anatomy, Faculty of Medicine, Ege University, Izmir, Turkey \\ ${ }^{2}$ Department of Internal Medicine, Faculty of Medicine, İzmir Katip Çelebi University, Izmir, Turkey
}

\begin{abstract}
Each kidney is supplied by a single renal artery, although renal artery variations are common. Variations of the renal artery have become important with the increasing number of renal transplantations. Numerous studies describe variations in renal artery anatomy. Especially the left renal artery is among the most critical arterial variations, because it is the referred side for resecting the donor kidney. During routine dissection in a formalin fixed male cadaver, we have found a bilateral renal artery variation. The presence of multiple renal arteries increases the complexity of the transplant surgery procedures.
\end{abstract}

Keywords: polar artery; renal artery variations; renal transplantation; testicular artery anatomy

Anatomy 2014;8:23-26, (c) 2014 TSACA

\section{Introduction}

As kidney transplantation improves the quality of a patient's life, renal artery anatomy is very important for surgeons. Although each kidney is supplied by a single renal artery, renal artery variations are common. ${ }^{[1-4]}$ In this study, we report a renal artery anomaly in an adult male cadaver.

\section{Case Report}

During dissection classes in the Department of Anatomy of Ege University, School of Medicine, a bilateral renal artery variation was found in a formalin fixed male cadaver without any trace of scars, adhesions or signs of trauma or operation. All measurements were taken with a machinist's caliper (FWP M-405-N-1), accurate to 0.1 $\mathrm{mm}$ and expressed in millimeters and centimeters. The study conforms to the provisions of the Helsinki Declaration of 1964 and all subsequent revisions. The surrounding adnexa were carefully examined for accompanying variations. The main right renal artery branched laterally from the aorta just below the origin of superior mesenteric artery, passing posterior to the inferior vena cava and the right renal vein. The right accessory artery supplied the lower renal pole (right lower polar artery). This right lower polar artery was arising from the abdominal aorta $1.2 \mathrm{~cm}$ below to the inferior mesenteric artery and crossed posterior to the ureter (Figures 1 and 3). On the left side, two renal hilar arteries were arising from abdominal aorta behind the left renal vein (Figures 2 and 3). The superior hilar artery divided into two branches after $0.6 \mathrm{~cm}$ of its origin. The left testicular artery originated from the inferior hilar artery (Figures 2 and 3$)$.

\section{Discussion}

Variations of the renal artery have become more clinically important with the increased frequency of renal transplantations. The presence of multiple renal arteries increases the complexity of the transplant surgery procedure. ${ }^{[47]}$ Numerous studies describe variations in renal artery anatomy. Especially the left renal artery anatomy is among most critical arterial variations because it is referred side for resecting the donor kidney. ${ }^{[6]}$

Extra renal arteries are divided into two types; polar types which supply kidney poles without passing through 
Figure 1. Polar artery (arrow) on the right side. ra: renal artery; rv: renal vein; u: ureter.

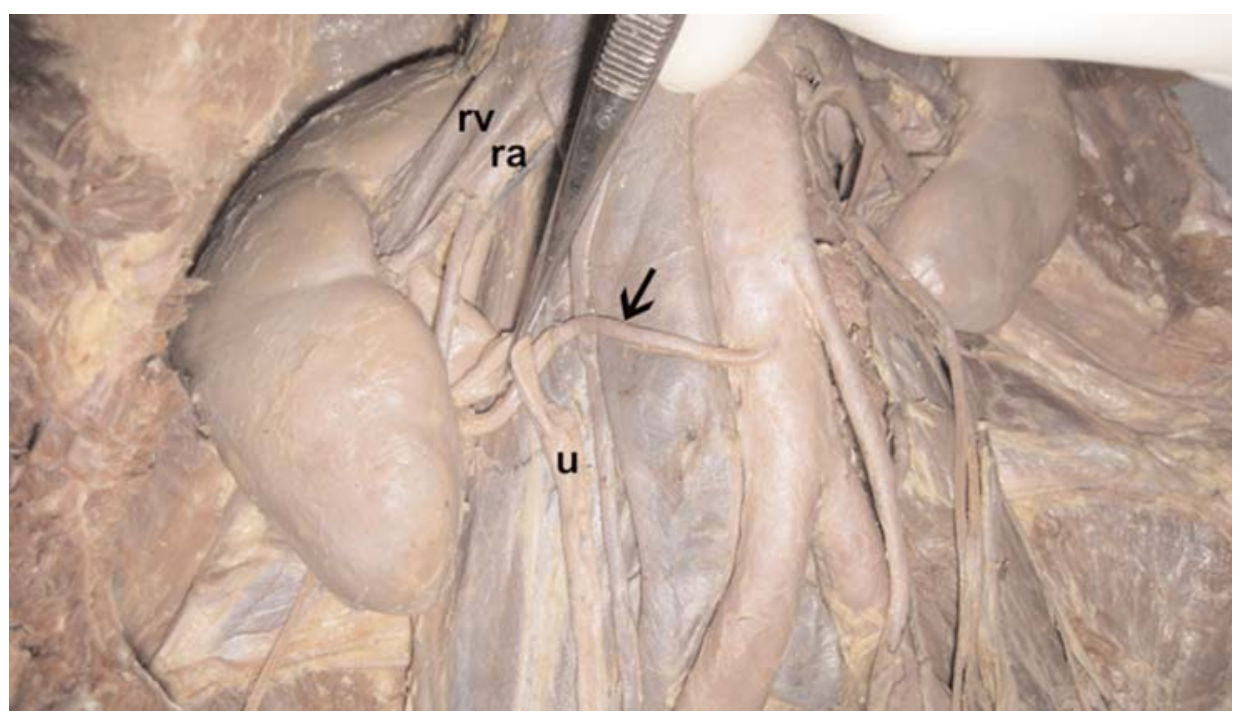

hilum, and accessory hilar types which supply the kidney after passing through the hilum. ${ }^{[8]}$

Accessory renal arteries are found frequently on the left side. ${ }^{[7]}$ Verma et al. ${ }^{[6]}$ discovered three renal arteries on the left side as a variation. The left main artery and two other arteries from a common trunk were found in their case. This was similar with the case in our report, except the superior renal artery had two branches in this case. ${ }^{[6]}$

Aristotle et al..$^{[1]}$ used 30 kidneys from 15 adult cadavers in their study. The normal pattern of blood supply was observed in $86.6 \%$ of their cases. They found an accessory renal artery in four cases (13.3\%). In three cadavers, lower polar arteries were dissected. In two cases, they were arising from abdominal aorta as found in the present case. ${ }^{[1]}$
The length of the right polar artery was $6.7 \mathrm{~cm}$ in our case. In some specimens, shorter polar renal arteries were determined. Chatzizacharias et al. ${ }^{[9]}$ emphasized the importance of the presence of multiple renal arteries, especially for short polar arteries. The short polar arteries enhance the degree of difficulty, due to segmental renal perfusion. Its injury may produce segmental ischemia with accompanying hypertension. ${ }^{[2,9]}$

Khamanarong et al. ${ }^{[2]}$ dissected 534 kidneys and presented the number and percent of renal arteries and polar arteries in six groups. We determined two of these groups on the left and right sides. On the right side, one hilar artery with one lower polar artery was observed in the present case. Khamanarong et al. ${ }^{[2]}$ determined this variation in $3 \%$ of the cases. Khamanarong et al. ${ }^{[2]}$ detected one
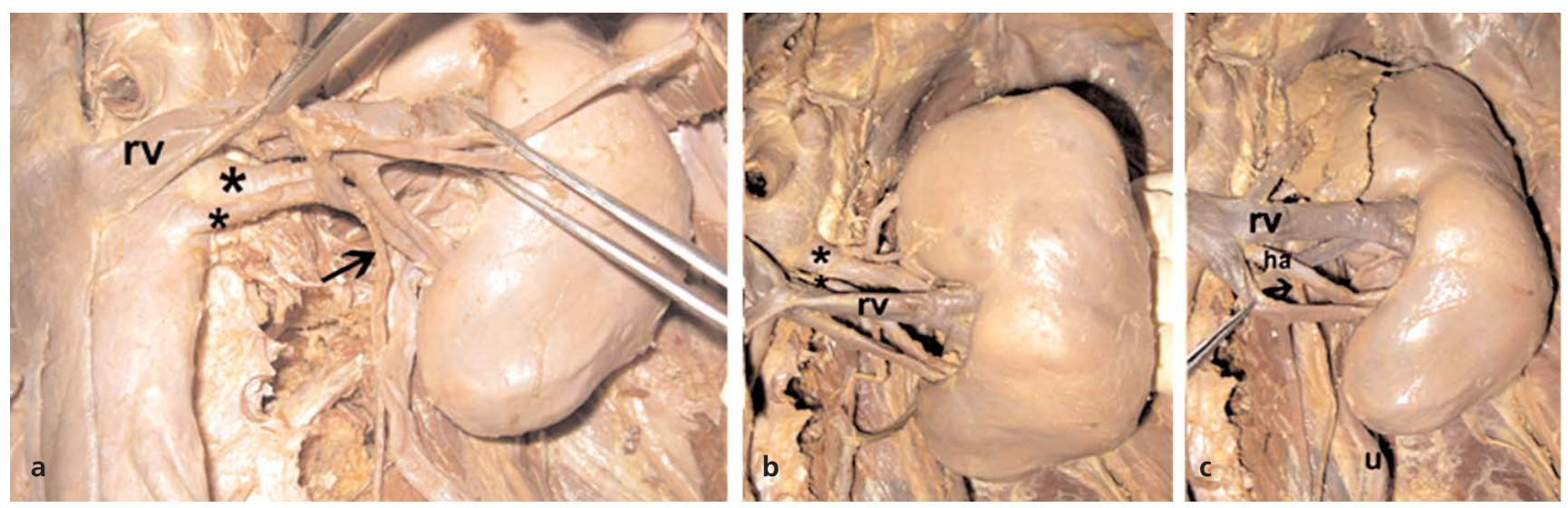

Figure 2. Two renal hilar arteries arising from abdominal aorta on the left side (a-c). Testicular artery (arrow) arising from inferior hilar artery on the left side. *: hilar arteries; ha: inferior hilar artery; rv: renal vein; u: ureter. 


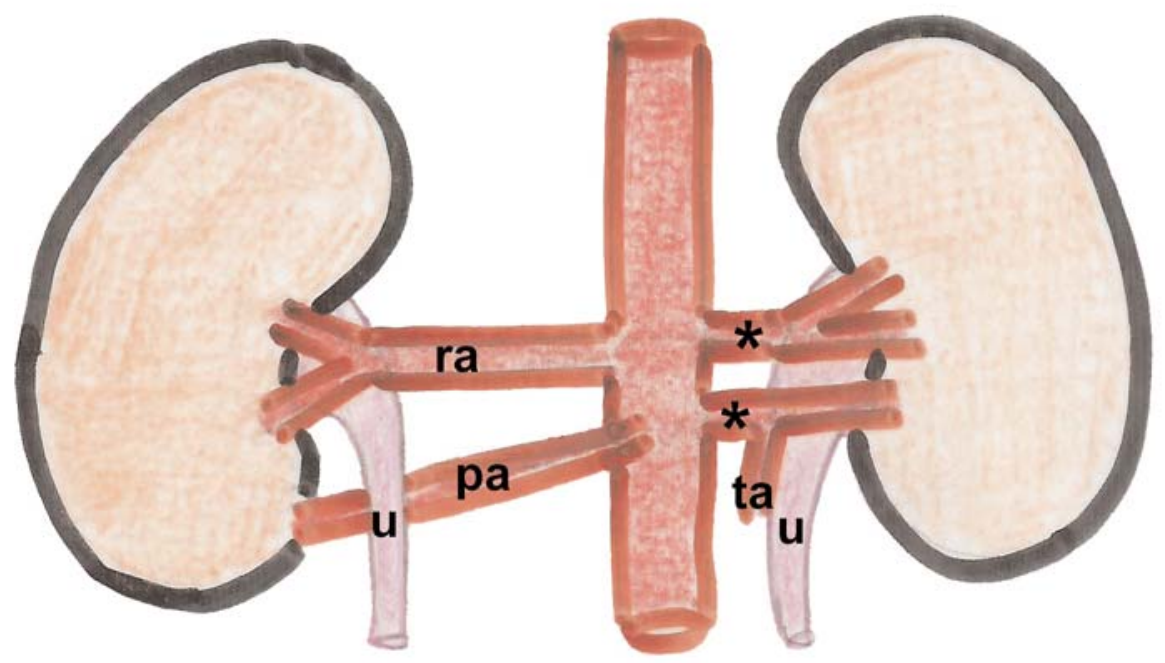

Figure 3. The schematic drawing of the case. *: hilar arteries; pa: polar artery; ra: renal artery; ta: testicular artery; u: ureter. lower polar artery posterior to ureter in three of 534 cases $(0.56 \%)$ as in the present case. Two of the three kidneys were on the right side as in the present case and one of these was on the left side.

Kumar et al. ${ }^{[10]}$ observed a bilateral vascular variations at the renal hilum. On the right side, renal artery with its normal origin and course from the abdominal aorta divided immediately into two branches. The superior branch pierced the upper pole of the kidney. On the left side, the hilum was wide and situated on the anterior surface of kidney. This bilateral renal hilar pattern with distorted shapes of kidneys was also not reported previously. ${ }^{[10]}$

In our case, two hilar arteries were found on the left side. Khamanarong et al. ${ }^{[2]}$ found the incidence of this variation $7.5 \%$. Shoja et al. ${ }^{[4]}$ observed eight cardinal peri-hilar renal artery branching patterns in their study on renal angiography of 81 kidneys. None of these patterns was similar with our specimen. ${ }^{[4]}$

The developing mesonephros, metanephros, suprarenal glands and gonads are supplied by nine pairs of lateral mesonephric arteries in the $18 \mathrm{~mm}$ fetus. They originate from the dorsal aorta. These arteries are divided into three groups as the cranial, middle and caudal groups. Persistence of more than one renal artery in the middle group results in multiple renal arteries. ${ }^{[7]}$

Parimala $^{[8]}$ reported bilateral aberrant renal arteries and a retroaortic left renal vein in an elderly male cadaver. This bilateral variation was very similar to the variation in our case. The left kidney was supplied with two renal arteries. There was a lower polar artery on the left side. Two renal arteries with different levels of origin from the aorta were observed also on the right side. ${ }^{[8]}$ Additionally, testicular artery arose from inferior hilar artery on the left side. Petru et al. ${ }^{[11]}$ assessed the gonadal arteries originating from the renal artery in 16 cases. This vascular variation also shows a significant importance for renal surgery and renal transplantation. A gonadal artery originating from an inferior hilar artery as demonstrated in the present case may be injured during the percutaneus treatment of pielo-ureteral junction. ${ }^{[11]}$

Chakravarthi $^{[12]}$ encountered an unilateral anomalous branching pattern of abdominal aorta during routine dissection of a male cadaver. A common trunk aroused $2.5 \mathrm{~cm}$ above the bifurcation of the abdominal aorta, gave off the inferior mesenteric artery, accessory renal artery to the left kidney and the left testicular artery. The embryogenesis of such multiple variations is not clear, but they have clinical significance for urologists, radiologists and surgeons. ${ }^{[12]}$

A precise knowledge of the renal artery variations is necessary for renal transplantation, renovascular hypertension, vascular reconstruction and reconstructive surgery for abdominal aortic aneurysms. The surgeons should keep in mind the possibility of these vascular anomalies. $^{[2,4,13,14]}$

\section{Conclusion}

Knowledge of the arterial pattern of the kidney is important for surgical procedures, especially for renal transplantation and nephrectomy to avoid any vascular complications.

\section{References}

1. Aristotle S, Sundarapandian, Felicia C. Anatomical study of variations in the blood supply of kidneys. J Clin Diagn Res 2013;7: 1555-7.

2. Khamanarong K, Prachaney $\mathrm{P}$, Utraravichien A, Tong-un T, Sripaoraya K. Anatomy of renal arterial supply. Clin Anat 2004; $17: 334-6$. 
3. Rani N, Singh S, Dhar P, Kumar R. Surgical importance of arterial segments of human kidneys: an angiography and corrosion cast study. J Clin Diagn Res 2014;8:1-3.

4. Shoja MM, Tubbs RS, Shakeri A, et al. Peri-hilar branching patterns and morphologies of the renal artery: a review and anatomical study. Surg Radiol Anat 2008;30:375-82.

5. Bakirtas H, Guvence N, Eroglu M, et al. Surgical approach to cases with multiple renal arteries in renal transplantation. Urol Int 2006;76:169-72.

6. Verma P, Arora AK, Sharma P, Mahajan A. Variations in branching pattern of renal artery and arrangement of hilar structures in the left kidney: clinical correlations, a case report. Ital J Anat Embryol 2012;117:118-22.

7. Budhiraja V, Rastogi R, Asthana AK. Renal artery variations: embryological basis and surgical correlation. Rom J Morphol Embryol 2010;51:533-6.

8. Parimala NB. Bilateral aberrant renal arteries with abnormal left renal vein: a case report. J Clin Diagn Res 2013;7:1425-6.
9. Chatzizacharias NA, Muthusami ASR, Sullivan M, Sinha S, Brockmann J. Use of gonadal vein interposition graft for implantation of polar artery in live donor renal transplantation. Transplantation 2010;90:1131-2.

10. Kumar N, Aithal AP, Guru A, Nayak SB. Bilateral vascular variations at the renal hilum: a case report. Case Rep Vasc Med 2012; 2012:968506. doi: 10.1155/2012/968506

11. Petru B, Elena Ş, Dan I, Constantin D. The morphology and the surgical importance of the gonadal arteries originating from the renal artery. Surg Radiol Anat 2007;29:367-71.

12. Chakravarthi KK. Unilateral multiple variations of renal, phrenic, suprarenal, inferior mesenteric and gonadal arteries. J Nat Sci Biol Med 2014;5:173-5.

13. Gandhi S, Singla RK, Suri RK, Mehta V, Rath G. Retroaortic left renal vein associated with unilateral superior renal polar artery: a clinico-embryological perspective. Clin Ter 2013;164:229-32.

14. Panagouli E, Tsaraklis A, Venieratos D. A lower polar additional renal artery in an ectopic intraperitoneal kidney. Folia Morphol (Warsz) 2011;70:56-8.

Correspondence to: Hülya Üçerler, MD

Department of Anatomy, Faculty of Medicine, Ege University, Izmir, Turkey

Phone: +90 2323903993 Fax: +90 2323903998

e-mail: hulyaucerler@gmail.com

Conflict of interest statement: No conflicts declared. 